\title{
AN ANSWER TO A CONJECTURE ON THE COUNTABLE PRODUCTS OF $k$-SPACES
}

\author{
HUAIPENG CHEN
}

(Communicated by James E. West)

\begin{abstract}
In this paper the author shows:
Theorem (CH). There is a $k_{\omega}$-space $X$ which is not locally compact but for which $X^{\omega}$ has a $k$-system.
\end{abstract}

This answers a conjecture of $Y$. Tanaka

\section{INTRODUCTION}

Let $\mathscr{C}$ be a compact covering of a space $X$. Then $X$ is said to have the weak topology with respect to $\mathscr{C}$ if $U \subset X$ is open (closed) in $X$ whenever $U \cap C$ is open (closed) in $C$ for each $C \in \mathscr{C}$. Following Arhangelskii [1] such a covering is called a $k$-system. We recall that a space $X$ is a $k$-space if it has the weak topology with respect to the cover consisting of all compact subsets of $X$. Then a space with a $k$-system is precisely a $k$-space. If $\mathscr{C}$ is point-countable (countable), $X$ is said to have a point-countable $k$-system (be a $k_{\omega}$-space). Recall a space $X$ has countable tightness $(t(X) \leq \omega)$ if whenever $x \in \bar{A}$, then $x \in \bar{C}$ for some countable $C \subset A$. Arhangelskii [2] has shown that $X^{\omega}$ has a $k$-system if $X$ is a locally compact space. Y. Tanaka has shown that if $X$ has a point-countable $k$-system and $t(X) \leq \omega$, then $X^{\omega}$ has a $k$-system if and only if $X$ is locally compact. In [3-5] Tanaka posed the following question: Let $X$ have a (point-) countable $k$-system. Then is $X$ locally compact if $X^{\omega}$ has a $k$-system? In this paper we give a negative answer under $(\mathrm{CH})$. Namely, under $(\mathrm{CH})$, there is a $k_{\omega}$-space $X$ which is not locally compact, but $X^{\omega}$ has a $k$-system.

\section{RESULTS}

For the ordinal $\omega_{2}+1$, let $Z$ be the topological sum of countably many copies of $\left[0, \omega_{2}\right]$ with the order topology. Then let $X$ be the quotient space obtained from $Z$ by identifying all the $\omega_{2}$ 's. Let $f: Z \rightarrow X$ be a canonical projection. Then $f$ is a closed mapping and $X$ is a $k_{\omega}$-space. Let $\mathscr{C}=$ $\left\{C_{n}=\bigcup_{i \leq n}\left[0, \omega_{2}\right]_{i} ; n<\omega\right\}$; then $\mathscr{C}$ is a countable $k$-system of $Z$. It

Received by the editors September 20, 1989 and, in revised form, March 21, 1990.

1991 Mathematics Subject Classification. Primary 54D50; Secondary 54B10.

Key words and phrases. $k$-space, $k$-system, locally compact, ordinal. 
will not cause confusion if we denote a $k$-system of $X$ by $\mathscr{C}$. Let $\mathscr{C}^{\omega}=$ $\left\{\prod_{i<\omega} C_{n_{i}} ; C_{n_{i}} \in \mathscr{C}\right\}$; then $\mathscr{C}^{\omega}$ is a $k$-system of the space $Z^{\omega}$ and $\mathscr{C}^{\omega}$ is a compact covering of the space $X^{\omega} . f: Z \rightarrow X$ is a continuous mapping, and so is $f^{\omega}=\prod_{i<\omega} f_{i}: Z^{\omega} \rightarrow X^{\omega}$, where $f_{i}=f$.

Lemma 1. Suppose $C$ is a compact subset in $X^{\omega}$ or $Z^{\omega}$. Then $X^{\omega}$ or $Z^{\omega}$ has a $\prod_{i<\omega} C_{n_{i}} \in \mathscr{C}^{\omega}$ such that $C \subset \prod_{i<\omega} C_{n_{i}}$.

Proof. By [4, Lemma 6].

Lemma 2. Let $f^{\omega}: Z^{\omega} \rightarrow X^{\omega}$ be a continuous mapping. For any $U \subset X$, $\left(f^{\omega}\right)^{-1}(U)$ is open in $Z^{\omega}$ iff $\left(\prod_{i<\omega} C_{n_{i}}\right) \cap U$ is open in $\prod_{i<\omega} C_{n_{i}}$ for each $\prod_{i<\omega} C_{n_{i}} \in \mathscr{C}^{\omega}$.

Proof. Suppose $\left(\prod_{i<\omega} C_{n_{i}}\right) \cap U$ is open in $\prod_{i<\omega} C_{n_{i}}$ for each $\prod_{i<\omega} C_{n_{i}} \in \mathscr{C}^{\omega}$ and $f^{\omega} \mid \prod_{i<\omega} C_{n_{i}}: \prod_{i<\omega} C_{n_{i}} \rightarrow \prod_{i<\omega} C_{n_{i}}$ is continuous. Let

$$
A=\left(f^{\omega} \mid \prod_{i<\omega} C_{n_{i}}\right)^{-1}\left[\left(\prod_{i<\omega} C_{n_{i}}\right) \cap U\right]=\left(f^{\omega}\right)^{-1}(U) \cap\left(\prod_{i<\omega} C_{\eta_{i}}\right) ;
$$

then $A$ is open in $\prod_{i<\omega} C_{n_{i}}\left(\subset Z^{\omega}\right)$. Since $Z^{\omega}$ has a $k$-system $\mathscr{C}^{\omega}$ by Lemma $1,(f)^{-1}(U)$ is open in $Z^{\omega}$.

Now assume $\left(f^{\omega}\right)^{-1}(U)$ is open in $Z^{\omega}$. Then $A$ is open in $\prod_{i<\omega} C_{n_{i}}$ for each $\prod_{i<\omega} C_{n_{i}}$. Therefore $\prod_{i<\omega} C_{n_{i}}-A$ is compact in $\prod_{i<\omega} C_{n_{i}}$, and so is $f^{\omega}\left[\prod_{i<\omega} C_{n_{i}}-A\right]=\prod_{i<\omega} C_{n_{i}}\left[\left[\left(\prod_{i<\omega} C_{n_{i}}\right) \cap U\right]\right.$. Then $U \cap\left(\prod_{i<\omega} C_{n_{i}}\right)$ is open in $\prod_{i<\omega} C_{n_{i}}$.

Theorem (CH). The space $X$ is a $k_{\omega}$-space which is not locally compact. But $X^{\omega}$ has a $k$-system.

Proof. By Lemma 1 it suffices to prove that given $U \subset X^{\omega}$, if $U \cap\left(\prod_{i<\omega} C_{n_{i}}\right)$ is open in $\prod_{i<\omega} C_{n_{i}}$ for each $\prod_{i<\omega} C_{n_{i}} \in \mathscr{C}^{\omega}$, then $U$ is open in $X^{\omega}$. Let $x \in U$; the general proof follows from the next three cases.

(A) $x=\left(\alpha_{i}\right), \alpha_{i} \neq \omega_{2}, i<\omega .\left(f^{\omega}\right)^{-1}\left(\alpha_{i}\right)=\left(\alpha_{i}\right) \in\left(f^{\omega}\right)^{-1}(U)$. By Lemma $2\left(f^{\omega}\right)^{-1}(U)$ is open in $Z^{\omega}$. Then $\left(\alpha_{i}\right)$ has a neighborhood $W=\left[\delta_{1}, \alpha_{1}^{\prime}\right] \times$ $\cdots \times\left[\delta_{n}, \alpha_{n}^{\prime}\right] \times Z \times Z \times \cdots$ in $Z^{\omega}$ such that $\left(\alpha_{i}\right) \in W \subset\left(f^{\omega}\right)^{-1}(U)$. Then $\left(\alpha_{i}\right) \in f^{\omega}(W)=\left[\delta_{1}, \alpha_{1}^{\prime}\right] \times \cdots \times\left[\delta_{n}, \alpha_{n}^{\prime}\right] \times X \times X \times \cdots \subset U$. Hence $U$ is a neighborhood of the point $x$ in $X^{\omega}$.

(B) $x=\left(\omega_{2}, \omega_{2}, \ldots\right)=\left(\omega_{2}\right)$. First we prove that there is an $N<\omega$ such that for each $\prod_{i<\omega} C_{n_{i}} \in \mathscr{C}^{\omega}$, there is a $\delta \in\left[0, \omega_{2}\right)$ with $\left(\bigcup_{i \leq n_{1}}\left[\delta, \omega_{2}\right]_{i}\right) \times$ $\left(\bigcup_{i \leq n_{2}}\left[\delta, \omega_{2}\right]_{i}\right) \times \cdots \times\left(\bigcup_{i \leq n_{N}}\left[\delta, \omega_{2}\right]_{i}\right) \times \prod_{i>N} C_{n_{i}} \subset U$.

Suppose that for each $n<\omega$ there is a $\prod_{i<\omega} C_{n_{i}} \in \mathscr{C}^{\omega}$ such that for each $\delta \in\left[0, \omega_{2}\right),\left[\left(\bigcup_{i \leq n_{1}}\left[\delta, \omega_{2}\right]_{i}\right) \times \cdots \times\left(\bigcup_{i \leq n_{n}}\left[\delta, \omega_{2}\right]_{i}\right) \times \prod_{i>n} C_{n_{i}}\right]-U \neq \varnothing$.

Then there is a $y_{\delta}=\left(\delta_{1}^{\prime}, \delta_{2}^{\prime}, \ldots, \delta_{n}^{\prime}, x_{\delta 1}, x_{\delta 2}, \ldots\right)$ such that

$$
y_{\delta} \in\left[\left(\bigcup_{i \leq n_{1}}\left[\delta, \omega_{2}\right]_{i}\right) \times \cdots \times\left(\bigcup_{i \leq n_{n}}\left[\delta, \omega_{2}\right]_{i}\right) \times \prod_{i>n} C_{n_{i}}\right]-U .
$$

Let $B_{n}=\left\{\left(\delta_{1}^{\prime}, \delta_{2}^{\prime}, \ldots, \delta_{n}^{\prime}, x_{\delta 1}, x_{\delta 2}, \ldots\right) ; \delta<\omega_{2}\right\}$. Then $B_{n} \cap U=\varnothing$ and $B_{n} \subset \prod_{i<\omega} C_{n_{i}}$. We show $\bar{B}_{n} \cap\left(\left\{\omega_{2}\right\} \times\left\{\omega_{2}\right\} \times \cdots \times\left\{\omega_{2}\right\} \times \prod_{i>n} C_{n_{i}}\right) \neq \varnothing$. In fact, suppose $\bar{B}_{n} \cap\left(\left\{\omega_{2}\right\} \times\left\{\omega_{2}\right\} \times \cdots \times\left\{\omega_{2}\right\} \times \prod_{i>n} C_{n_{i}}\right)=\varnothing$. Since $\bar{B}_{n}$ and $\left\{\omega_{2}\right\} \times\left\{\omega_{2}\right\} \times \cdots \times\left\{\omega_{2}\right\} \times \prod_{i>n} C_{n_{i}}$ are compact in $\prod_{i<\omega} C_{n_{i}},\left\{\omega_{2}\right\} \times \cdots \times\left\{\omega_{2}\right\} \times$ 
$\prod_{i>n} C_{n_{i}}$ has a neighborhood $W=\left(\bigcup_{i \leq n_{1}}\left[\delta_{0}, \omega_{2}\right]_{i}\right) \times \cdots \times\left(\bigcup_{i \leq n_{n}}\left[\delta_{0}, \omega_{2}\right]_{i}\right) \times$ $\prod_{i>n} C_{n_{i}}$ in $\prod_{i<\omega} C_{n_{i}}$ such that $W \cap \bar{B}_{n}=\varnothing$. On the other hand, we let

$$
A_{n}=\left\{\left(\delta_{1}^{\prime}, \delta_{2}^{\prime}, \ldots, \delta_{n}^{\prime}, x_{\delta 1}, x_{\delta_{2}}, \ldots\right) \in B_{n} ; \delta_{0} \leq \delta<\omega_{2}\right\}
$$

then $A_{n} \neq \varnothing, A_{n} \subset B_{n}$, and $A_{n} \subset W$. Therefore, $A_{n}=A_{n} \cap B_{n} \subset W \cap \bar{B}_{n}=\varnothing$, a contradiction.

Hence there is a $\left(\omega_{2}, \omega_{2}, \ldots, \omega_{2}, x_{n 1}, x_{n 2}, \ldots\right) \in \bar{B}_{n}$. Since $\left(\prod_{i<\omega} C_{n_{i}}\right) \cap$ $U$ is open in $\prod_{i<\omega} C_{n_{i}},\left(\omega_{2}, \omega_{2}, \ldots, \omega_{2}, x_{n 1}, x_{n 2}, \ldots\right) \in \bar{B}_{n}$ and $\bar{B}_{n} \subset$ $\prod_{i<\omega} C_{n_{i}}-\left[\left(\prod_{i<\omega} C_{n_{i}}\right) \cap U\right]$; hence, $\left(\omega_{2}, \omega_{2}, \ldots, \omega_{2}, x_{n 1}, x_{n 2}, \ldots\right) \notin U$. Let

$$
\begin{aligned}
B=\left\{\left(\omega_{2}, x_{11}, x_{12}, \ldots\right),\left(\omega_{2}, \omega_{2},\right.\right. & \left.x_{21}, x_{22}, \ldots\right), \ldots, \\
& \left.\left(\omega_{2}, \ldots, \omega_{2}, x_{n 1}, \ldots\right), \ldots\right\} ;
\end{aligned}
$$

then $B \cap U=\varnothing . \prod_{1}(B)=\left\{\omega_{2}\right\}, \prod_{i+1}(B)=\left\{\omega_{2}, x_{1 i}, x_{2 i-1}, \ldots, x_{i 1}\right\}, i=$ $1,2, \ldots$. For each $i<\omega, \prod_{i}(B)$ is finite in $X$, so there is a $C_{m_{i}} \in \mathscr{C}$ such that $B \subset \prod_{i<\omega} C_{m_{i}}$. Since $U \cap\left(\prod_{i<\omega} C_{m_{i}}\right)$ is open in $\prod_{i<\omega} C_{m_{i}}$, we have $\bar{B} \subset$ $\prod_{i<\omega} C_{m_{i}}-\left[\left(\prod_{i<\omega} C_{m_{i}}\right) \cap U\right]$. This $B$ is a sequence converging to $\left(\omega_{2}, \omega_{2}, \ldots\right)$ in $\prod_{i<\omega} C_{m_{i}}$. Indeed, take a neighborhood $W=\left(\bigcup_{i \leq m_{1}}\left[\delta, \omega_{2}\right]_{i}\right) \times \cdots \times$ $\left(\bigcup_{i \leq m_{n}}\left[\delta, \omega_{2}\right]_{i}\right) \times \prod_{i>n} C_{m_{i}}$ of $\left(\omega_{2}, \omega_{2}, \ldots\right)$ in $\prod_{i<\omega} C_{m_{i}}$. Then $\left\{\left(\omega_{2}, \ldots\right.\right.$, $\left.\left.\omega_{2}, x_{n+1,1}, x_{n+1,2}, \ldots\right),\left(\omega_{2}, \ldots, \omega_{2}, x_{n+2,1}, x_{n+2,2}, \ldots\right), \ldots\right\} \subset W$. Therefore, $\left(\omega_{2}, \omega_{2}, \ldots\right) \in \bar{B} \subset \prod_{i<\omega} C_{m_{i}}-\left[\left(\prod_{i<\omega} C_{m_{i}}\right) \cap U\right],\left(\omega_{2}\right) \notin U$. It is a contradiction with $\left(\omega_{2}\right) \in U$. Second we look for a neighborhood $W$ of $\left(\omega_{2}\right)$ in $X^{\omega}$ such that $\left(\omega_{2}\right) \in W \subset U$. Let $\mathscr{C}^{\omega}=\left\{\prod_{i<\omega} C_{n_{i}} ; C_{n_{i}} \in\right.$ $\mathscr{C}\}=\left\{K_{\alpha} ; \alpha<\omega_{1}\right\}$. By the previous arguments, there is an $N<\omega$ such that for each $K_{\alpha}=\prod_{i<\omega} C_{n_{i}}$ there is a $\delta_{\alpha}<\omega_{2}$ such that $\left(\bigcup_{i \leq n_{1}}\left[\delta_{\alpha}, \omega_{2}\right]_{i}\right) \times$ $\cdots \times\left(\bigcup_{i<n_{N}}\left[\delta_{\alpha}, \omega_{2}\right]_{i}\right) \times \prod_{i>N} C_{n_{i}} \subset U$. Let $\delta=\sup _{\alpha<\omega_{1}} \delta_{\alpha} ;$ then $\delta<\omega_{2}$ and $\left[\left(\bigcup_{i \leq n_{1}}\left[\delta, \omega_{2}\right]_{i}\right) \times \cdots \times\left(\bigcup_{i \leq n_{N}}\left[\delta, \omega_{2}\right]_{i}\right) \times \prod_{i>N} C_{n_{i}} \subset U\right.$ for any $\prod_{i<\omega} C_{n_{i}} \in \mathscr{C}^{\omega}$. Thus let $V=\bigcup_{i<\omega}\left[\delta, \omega_{2}\right]_{i}$ be a neighborhood of the point $\left(\omega_{2}\right)$ in $X$; then

$$
\begin{aligned}
\bigcup_{\alpha<\omega_{1}} & {\left[\left(\bigcup_{i \leq n_{1}}\left[\delta, \omega_{2}\right]_{i}\right) \times \cdots \times\left(\bigcup_{i \leq n_{N}}\left[\delta, \omega_{2}\right]_{i}\right) \times \prod_{i>N} C_{n_{i}}\right.} \\
= & V \times \cdots \times V \times X \times X \times \cdots=W \subset U .
\end{aligned}
$$

(C) $x=\left(\alpha_{1}, \omega_{2}, \alpha_{2}, \omega_{2}, \ldots, \alpha_{n}, \omega_{2}, \alpha_{n+1}, \omega_{2}, \ldots\right), \alpha_{i} \neq \omega_{2}$. First we show that there is an $N<\omega$ and a $\delta_{i} \in\left(0, \alpha_{i}\right), i=1,2, \ldots, N$, such that for each $\delta_{i}^{\prime} \in\left(\delta_{i}, \alpha_{i}\right], i=1,2, \ldots, N$, there is a $V \in \mathscr{U}$ with $\left\{\delta_{1}^{\prime}\right\} \times$ $V \times\left\{\delta_{2}^{\prime}\right\} \times V \times \cdots \times\left\{\delta_{n}^{\prime}\right\} \times V \times X \times X \times \cdots \subset U$, where $\mathscr{U}=\left\{V_{\alpha}=\right.$ $\left.\bigcup_{i<\omega}\left[\alpha, \omega_{2}\right]_{i} ; \alpha<\omega_{2}\right\}$. Suppose for each $n<\omega$ and each $\delta_{i} \in\left(0, \alpha_{i}\right), i=$ $1,2, \ldots, n$, there is a $\delta_{i}^{\prime} \in\left(\delta_{i}, \alpha_{i}\right], i=1,2, \ldots, n$, such that for each $V \in \mathscr{U},\left(\left\{\delta_{1}^{\prime}\right\} \times V \times\left\{\delta_{2}^{\prime}\right\} \times V \times \cdots \times\left\{\delta_{n}^{\prime}\right\} \times V \times X \times X \times \cdots\right)-U \neq \varnothing$. Take any $\delta_{i} \in\left(0, \alpha_{i}\right), i<\omega$. For each $n<\omega$ and each $\delta_{i} \in\left(0, \alpha_{i}\right), i=$ $1,2, \ldots, n$, there is a $\delta_{i}^{\prime} \in\left(\delta_{i}, \alpha_{i}\right], i=1,2, \ldots, n$, such that for each $V \in \mathscr{U}$ there is an $x_{v}=\left(\delta_{n 1}, \alpha_{v 1}, \delta_{n 2}, \alpha_{v 2}, \ldots, \delta_{n n}, \alpha_{v n}, x_{v 1}, x_{v 2}, \ldots\right)$ such that $x_{v} \in\left(\left\{\delta_{1}^{\prime}\right\} \times V \times\left\{\delta_{2}^{\prime}\right\} \times V \times \cdots \times\left\{\delta_{n}^{\prime}\right\} \times V \times X \times X \times \cdots\right)-U$. Let $B_{n}=\left\{x_{v}=\left(\delta_{n 1}, \alpha_{v 1}, \delta_{n 2}, \alpha_{n 2}, \ldots, \delta_{n n}, \alpha_{v n}, x_{v 1}, x_{v 2}, \ldots\right) ; V \in \mathscr{U}\right\}$. There are two possible cases.

Case 1. $\left|B_{n}\right|<\aleph_{2}$. By $|\mathscr{U}|=\aleph_{2}$ there is an $x_{v^{\prime}}$ which repeats at least $\aleph_{2}$ times in $B_{n}$. Then the point $x_{v^{\prime}}=\left(\beta_{1}, \omega_{2}, \beta_{2}, \omega_{2}, \ldots, \beta_{n}, \omega_{2}, x_{n 1}, x_{n 2}, \ldots\right)$, 
$x_{n_{i}} \in X, i<\omega$, and $\beta_{i} \in\left(\delta_{i}, \alpha_{i}\right], i=1,2, \ldots, n$. If some $2 k$ th $(k \leq n)$ coordinate of $x_{v^{\prime}}$ is not $\omega_{2}$, then let this $2 k$ th $(k \leq n)$ coordinate be $\alpha$ and $\mathscr{U}_{1}=\left\{V \in \mathscr{U} ; V \supset V_{\alpha+1}\right\}$. Since $\mathscr{U}$ is linearly ordered by inclusion, $\left|\mathscr{U}_{1}\right| \leq \aleph_{1}$; therefore, $x_{v^{\prime}}$ repeats at most $\aleph_{1}$ times in $\left\{x_{v} \in B_{n} ; V \in \mathscr{U}_{1}\right\}$. Hence there is a $\delta \geq \alpha+1$ such that $x_{v_{\delta}}=x_{v^{\prime}}$. On the other hand, if $\alpha \in V_{\delta}=\bigcup_{i<\omega}\left[\delta, \omega_{2}\right]_{i}$, then $\alpha \geq \delta \geq \alpha+1$, which is a contradiction. By the $x_{v^{\prime}} \in B_{n}$ and $B_{n} \cap U=\varnothing$, it follows that $x_{v^{\prime}} \notin U$.

Case 2. $\left|B_{n}\right|=\aleph_{2}$. Since $\left|\left\{\prod_{i<\omega} C_{n_{i}} ; C_{n_{i}} \in \mathscr{C}\right\}\right|=\aleph_{1}$, there is a $\prod_{i<\omega} C_{n_{i}} \in$ $\mathscr{C}^{\omega}$ such that $\left|\left(\prod_{i<\omega} C_{n_{i}}\right) \cap B_{n}\right|=\aleph_{2}$. Let $\mathscr{U}_{1}=\left\{V \in \mathscr{U} ; x_{v} \in B_{n} \cap\left(\prod_{i<\omega} C_{n_{i}}\right)\right\}$; then $\mathscr{U}_{1}$ is confinal in $\mathscr{U}$. Let $A_{n}=\left\{x_{v} \in B_{n} \cap\left(\prod_{i<\omega} C_{n_{i}}\right) ; V \in \mathscr{U}_{1}\right\}$ (hence $\left.A_{n} \subset \prod_{i<\omega} C_{n_{i}}\right)$ and $K=\left\{\alpha_{1}\right\} \times\left\{\omega_{2}\right\} \times\left\{\alpha_{2}\right\} \times\left\{\omega_{2}\right\} \times \cdots \times\left\{\alpha_{n}\right\} \times\left\{\omega_{2}\right\} \times$ $\prod_{i>2 n} C_{n_{i}}$; then we will show that $\bar{A}_{n} \cap K \neq \varnothing$. Indeed, suppose $\bar{A}_{n} \cap K=$ $\varnothing$, take a neighborhood $W=\left[\beta_{1}^{\prime}, \alpha_{1}^{\prime}\right] \times\left(\bigcup_{i \leq n_{2}}\left[\delta, \omega_{2}\right]_{i}\right) \times \cdots \times\left[\beta_{n}^{\prime}, \alpha_{n}^{\prime}\right] \times$ $\left(\bigcup_{i \leq n_{2 n}}\left[\delta, \omega_{2}\right]_{i}\right) \times \prod_{i>2 n} C_{n_{i}}$ of $K$ in $\prod_{i<\omega} C_{n_{i}}$ such that $W \cap \bar{A}_{n}=\varnothing$. Let $A_{n}^{*}=\left\{x_{v} \in A_{n} ; V \subset V_{\delta}=\bigcup_{i<\omega}\left[\delta, \omega_{2}\right]_{i}\right\}$; then $A_{n}^{*} \subset W$ and $\left|A_{n}^{*}\right|=\aleph_{2}$. On the other hand, $A_{n}^{*} \subset A_{n}$, so $A_{n}^{*}=A_{n}^{*} \cap A_{n} \subset W \cap A_{n}=\varnothing$, a contradiction. Thus there is an $x_{n_{i}} \in C_{n_{2 n+i}}(i<\omega)$ such that

$$
\begin{gathered}
\left(\alpha_{1}, \omega_{2}, \alpha_{2}, \omega_{2}, \ldots, \alpha_{n}, \omega_{2}, x_{n 1}, x_{n 2}, \ldots\right) \\
\in \bar{A}_{n} \subset \prod_{i<\omega} C_{n_{i}}-\left[\left(\prod_{i<\omega} C_{n_{i}}\right) \cap U\right] .
\end{gathered}
$$

Hence $\left(\alpha_{1}, \omega_{2}, \alpha_{2}, \omega_{2}, \ldots, \alpha_{n}, \omega_{2}, x_{n 1}, x_{n 2}, \ldots\right) \notin U$. Therefore, for either Case 1 or 2 , there is a $\delta_{n_{i}} \in\left(\delta_{i}, \alpha_{i}\right], i=1,2, \ldots, n$, such that

$$
y_{n}=\left(\delta_{n 1}, \omega_{2}, \delta_{n 2}, \omega_{2}, \ldots, \delta_{n n}, \omega_{2}, x_{n 1}, x_{n 2}, \ldots\right) \notin U .
$$

Let $B=\left\{y_{n} ; n=1,2, \ldots\right\}$. Then $\prod_{1}(B)=\left\{\delta_{1+i, 1} ; i<\omega\right\}, \prod_{2}(B)=$ $\left\{\omega_{2}\right\}, \prod_{2 n+1}(B)=\left\{x_{1,2 n-1}, x_{2,2 n-3}, \ldots, x_{n, 1}\right\} \cup\left\{\delta_{n+1+i, n+1} ; i<\omega\right\}, n=$ $\left.1,2, \ldots, \prod_{2 n}(B)=x_{1,2 n-2}, x_{2,2 n-4}, \ldots, x_{n-1,2}\right\} \cup\left\{\omega_{2}\right\}, n=2,3, \ldots$, and $B \cap U=\varnothing$. Obviously, for each $i<\omega$ there is a $C_{m_{i}} \in \mathscr{C}$ such that $\prod_{i}(B) \subset C_{m_{i}}$; therefore, $B \subset \prod_{i<\omega} C_{m_{i}}$. Let $K=\left(\delta_{1}, \alpha_{1}\right] \times\left\{\omega_{2}\right\} \times \cdots \times$ $\left(\delta_{n}, \alpha_{n}\right] \times\left\{\omega_{2}\right\} \times\left(\delta_{n+1}, \alpha_{n+1}\right] \times\left\{\omega_{2}\right\} \times \cdots ;$ then $K \cap \bar{B} \neq \varnothing$. In fact, suppose $\bar{B} \cap K=\varnothing$, where we assume $K \subset \prod_{i<\omega} C_{m_{i}}$. Then there is a neighborhood $W=\left[B_{1}, \alpha_{1}\right] \times\left(\bigcup_{i \leq m_{2}}\left[\delta, \omega_{2}\right]_{i}\right) \times \cdots \times\left[\beta_{n}, \alpha_{n}\right] \times\left(\bigcup_{i \leq m_{n}}\left[\delta, \omega_{2}\right]_{i}\right) \times$ $\prod_{i>n} C_{m_{i}}$ of $K$ in $\prod_{i<\omega} C_{m_{i}}$ such that $W \cap \bar{B}=\varnothing$. On the other hand, $\varnothing \neq\left\{y_{n}, y_{n+1}, \ldots\right\} \subset B \cap W=\varnothing$, a contradiction. Take any $x \in K \cap$ $\bar{B} \neq \varnothing, x=\left(\delta_{1}^{\prime}, \omega_{2}, \delta_{2}^{\prime}, \omega_{2}, \ldots, \delta_{n}^{\prime}, \omega_{2}, \ldots\right)$. By $U \cap\left(\prod_{i<\omega} C_{m_{i}}\right)$ being open in $\prod_{i<\omega} C_{m_{i}}$ and $B \subset \prod_{i<\omega} C_{m_{i}}-\left[\left(\prod_{i<\omega} C_{m_{i}}\right) \cap U\right]$, it follows that $\left(\delta_{1}^{\prime}, \omega_{2}, \ldots, \delta_{n}^{\prime}, \omega_{2}, \delta_{n+1}^{\prime}, \omega_{2}, \ldots\right) \in \bar{B} \subset \prod_{i<\omega} C_{m_{i}}-\left[\left(\prod_{i<\omega} C_{m_{i}}\right) \cap U\right]$. Therefore, $\left(\delta_{1}^{\prime}, \omega_{2}, \ldots, \delta_{n}^{\prime}, \omega_{2}, \delta_{n+1}^{\prime}, \omega_{2}, \ldots\right) \notin U$. Hence it is proven that for each $\delta_{i} \in\left(0, \alpha_{i}\right), i<\omega$, there is a $\delta_{i}^{\prime} \in\left(\delta_{i}, \alpha_{i}\right], i<\omega$, such that $\left(\delta_{1}^{\prime}, \omega_{2}, \ldots\right.$, $\left.\delta_{n}^{\prime}, \omega_{2}, \delta_{n+1}^{\prime}, \omega_{2}, \ldots\right) \notin U$. Let

$$
A=\left\{\left(\delta_{1}^{\prime}, \omega_{2}, \ldots, \delta_{n}^{\prime}, \omega_{2}, \ldots\right) ; \delta_{i}^{\prime} \in\left(0, \alpha_{i}\right), \delta_{i}^{\prime} \in\left(\delta_{i}, \alpha_{i}\right], i<\omega\right\} ;
$$

then $A \cap U=\varnothing$ and $A \subset K_{1}=\left[0, \alpha_{1}\right] \times\left\{\omega_{2}\right\} \times \cdots \times\left[0, \alpha_{n}\right] \times\left\{\omega_{2}\right\} \times$ $\left[0, \alpha_{n+1}\right] \times\left\{\omega_{2}\right\} \times \cdots$. By $U \cap K_{1}$ being open in $K_{1}$, it follows that $\left(\alpha_{1}, \omega_{2}, \ldots, \alpha_{n}, \omega_{2}, \ldots\right) \in \bar{A} \subset K_{1}-\left(K_{1} \cap U\right)$. This contradicts the fact 
$\left(\alpha_{1}, \omega_{2}, \ldots, \alpha_{n}, \omega_{2}, \alpha_{n+1}, \omega_{2}, \ldots\right) \in U$. Second, we look for a neighborhood $W$ of $\left(\alpha_{1}, \omega_{2}, \ldots, \alpha_{n}, \omega_{2}, \ldots\right)$ in $X^{\omega}$ such that $W \subset U$. We already showed that there is an $N<\omega$ and a $\delta_{i} \in\left(0, \alpha_{i}\right), i=1,2, \ldots, N$, such that for each $\delta_{i}^{\prime} \in\left(\delta_{i}, \alpha_{i}\right], i=1,2, \ldots, N$, there exists a $V \in \mathscr{U}$ with $\left\{\delta_{1}^{\prime}\right\} \times V \times\left\{\delta_{2}^{\prime}\right\} \times V \times \cdots \times\left\{\delta_{N}^{\prime}\right\} \times V \times X \times X \times \cdots \subset U$. On the other hand,

$$
\left|\left[0, \alpha_{1}\right] \times\left[0, \alpha_{2}\right] \times \cdots \times\left[0, \alpha_{n}\right]\right| \leq \aleph_{1}<\aleph_{2} .
$$

Then for each $\left(\delta_{1}^{\prime}, \delta_{2}^{\prime}, \ldots, \delta_{N}^{\prime}\right) \in\left(\delta_{1}, \alpha_{1}\right] \times \cdots \times\left(\delta_{N}, \alpha_{N}\right]$, there is an $\alpha<\omega_{2}$ such that $\left\{\delta_{1}^{\prime}\right\} \times V_{\alpha} \times \cdots \times\left\{\delta_{N}^{\prime}\right\} \times V_{\alpha} \times X \times X \times \cdots \subset U$. Let $\delta=\sup _{\alpha<\omega_{1}} \alpha$. Then $\delta<\omega_{2}, V_{\delta} \in \mathscr{U}$, and for each $\left(\delta_{1}^{\prime}, \delta_{2}^{\prime}, \ldots, \delta_{N}^{\prime}\right) \in\left(\delta_{1}, \alpha_{1}\right] \times \cdots \times\left(\delta_{N}, \alpha_{N}\right]$, $\left\{\delta_{1}^{\prime}\right\} \times V_{\delta} \times \cdots \times\left\{\delta_{N}^{\prime}\right\} \times V_{\delta} \times X \times X \times \cdots \subset U$. Therefore, $\left(\delta_{1}, \alpha_{1}\right] \times V_{\delta} \times \cdots \times$ $\left(\delta_{N}, \alpha_{N}\right] \times V_{\delta} \times X \times X \times \cdots \subset U$.

(D) In general, if $x \in U$, we can show that $U$ is a neighborhood of $x$ in $X^{\omega}$ by using the technique of (B) or (C).

Remark. In the previous theorem, we can replace "not locally compact" by "not even a $k^{\prime}$-space". Indeed, let $Z_{0}=\left[1, \omega_{0}\right], Z_{n}=\left[0, \omega_{2}\right]_{n}$ for $n=1,2, \ldots$, with order topology. Let $Z$ be the topological sum of $Z_{n}, i<\omega$; then $Z$ is a locally compact Hausdorff space. Let $Y$ be the quotient space obtained from $Z$ by identifying each " $n$ " in $Z_{0}$ and " $\omega_{2}$ " in $Z_{n}$ for $n=1,2, \ldots$. Then $Y$ is a $k_{\omega}$-space but not a $k^{\prime}$-space. In fact, $\omega_{0} \in \overline{\bigcup_{i<\omega}\left[0, \omega_{2}\right)_{i}}$ but $\omega_{0} \notin \overline{K \cap\left(\bigcup_{n<\omega}\left[0, \omega_{2}\right)_{n}\right)}$ for each compact subset $K \subset Y$. Let $g: Y \rightarrow$ $Y /\left[1, \omega_{0}\right]=X$ be a canonical projection. Then $g$ is a prefect mapping, as is $g^{\omega}$. Since $X^{\omega}$ has a $k$-system, so does $Y^{\omega}$. Then $Y$ has the desired property.

\section{ACKNOWLEDGMENT}

The author wishes to thank Y. Tanaka for his suggestions.

\section{REFERENCES}

1. A. V. Arhangelskii, Factor mapping of metric spaces, Soviet Math. Dokl. 5 (1964), 368-371.

2. $\frac{}{1-62}$. Bicompact sets and the topology of spaces, Trans. Moscow Math. Soc. 13 (1965),

3. Y. Tanaka, Some necessary conditions for products of spaces, Bull. Tokyo Gakugei Univ. 30 (1978), 1-16.

4. __ Point-countable $k$-system and products of k-spaces, Pacific J. Math. 101 (1982), 199-208.

5. __ On the products of $k$-spaces question, Q\&A in General Topology 1 (1983).

Department of Mathematics, Qinghai Normal University, Xining, Qinghai, People's REPUBLIC OF CHINA

Current address: c/o Professor Takao Hoshina, Institute of Mathematics, University of Tsukuba, Tsukuba-shi, Ibaraki 305, Japan 